PROCEEDINGS OF THE

AMERICAN MATHEMATICAL SOCIETY

Volume 135, Number 3, March 2007, Pages 689-693

S 0002-9939(06)08544-3

Article electronically published on September 11, 2006

\title{
INTERSECTIONS OF CONJUGACY CLASSES AND SUBGROUPS OF ALGEBRAIC GROUPS
}

\author{
ROBERT M. GURALNICK
}

(Communicated by Lance W. Small)

\begin{abstract}
We show that if $H$ is a reductive group, then $n$th roots of conjugacy classes are a finite union of conjugacy classes, and that if $G$ is an algebraic overgroup of $H$, then the intersection of $H$ with a conjugacy class of $G$ is a finite union of $H$-conjugacy classes. These results follow from results on finiteness of unipotent classes in an almost simple algebraic group.
\end{abstract}

\section{INTRODUCTION}

Let $G$ be a reductive algebraic group over an algebraically closed field $k$ (i.e. $G$ has no normal unipotent subgroup of positive dimension). Note that $G$ need not be connected and is reductive if and only if its connected component containing 1 is reductive.

Conjugacy classes of unipotent elements in algebraic groups with simple connected components are reasonably well understood (even in the disconnected case). In particular, it is known that there are only finitely many conjugacy classes of such elements.

The point of this article is to use this to deduce some interesting consequences about conjugacy classes in reductive groups. Our main results are:

Theorem 1.1. Let $G$ be a reductive algebraic group over an algebraically closed field $k$. Let $C$ be a conjugacy class in $G$ and $n$ a positive integer. Then $D:=\{x \in$ $\left.G \mid x^{n} \in C\right\}$ is a finite union of conjugacy classes. In particular, $G$ has only finitely many conjugacy classes of elements of order $n$.

It is well known that there are only finitely many conjugacy classes of elements of order $n$ in a connected reductive group. See [2.

Theorem 1.2. Let $G$ be an algebraic group over an algebraically closed field $k$. If $H$ is a reductive subgroup of $G$ and $C$ is a conjugacy class of $G$, then $C \cap H$ is a finite union of $H$-conjugacy classes.

An immediate consequence is a result about dimensions of intersections of subgroups and conjugacy classes.

Received by the editors October 11, 2005.

2000 Mathematics Subject Classification. Primary 20G15.

Key words and phrases. Conjugacy classes, algebraic groups, reductive groups, $n$th roots.

The author gratefully acknowledges the support of NSF grant DMS 0140578. He also thanks Ben Martin, Gerhard Röhrle and Daniel Goldstein for helpful comments, and the IAS for its support.

(C)2006 American Mathematical Society Reverts to public domain 28 years from publication 
Corollary 1.3. Let $C$ be a conjugacy class of the algebraic group $G$. Let $H$ be a reductive subgroup of $G$. Then $\operatorname{dim}(C \cap H)=\operatorname{dim} D$ for some $H$-conjugacy class $D \subseteq C \cap H$.

These results are known in many cases. Once we have the result about unipotent conjugacy classes, it is not difficult to deduce these results.

Of course, these results are false for nonreductive groups (for example take $G$ to be a positive-dimensional unipotent group). It does hold in some cases for parabolic subgroups of reductive groups, but not always (see [6] for examples). Ben Martin and Gerhard Röhrle have asked whether there is an analog of Theorem 1.2 for $H$ a reductive subgroup of $G$ acting on $G^{n}$ for $n>1$ (i.e. $G$ acts on $G^{n}$ by simultaneous conjugation - if $X$ is a $G$-orbit in $G^{n}$, when is $X \cap H^{n}$ a finite union of $H$-orbits?). This has a positive answer in certain cases (for example, by Weil this is true for finite subgroups of order coprime to the characteristic, and somewhat more generally by results of Slodowy [8]). See also [5] and [1].

The paper is organized as follows. In the next section, we gather some preliminary results, and in the third section we prove Theorems 1.1 and 1.2 We give the proofs for algebraic groups over an algebraically closed field $k$ of characteristic $p>0$. The proofs in characteristic zero are easier but slightly different. One can also deduce the characteristic zero results from the positive characteristic results.

\section{Preliminary Results}

Let $k$ be an algebraically closed field of characteristic $p>0$. All algebraic groups are over the field $k$. If $H$ is an algebraic group, we let $H^{0}$ denote the connected component of $H$ containing 1 .

The following results are crucial for our results. See the book by Spaltenstein [10. See also Lusztig [3] and Sorlin [9]. Note that the disconnected case essentially occurs only for $p=2$ or 3 . Lusztig [3] proved the result in the connected case. Richardson [7] had earlier proved the result in many cases.

Theorem 2.1. Let $S$ be a simple algebraic group. There are only finitely many conjugacy classes of unipotent elements in $S$.

Theorem 2.2. Let $S$ be a simple algebraic group of characteristic $p$. Let $S \leq G \leq$ $\operatorname{Aut}(S)$. If $x \in G \backslash S$ has order $p^{a}$, then there are only finitely many $S$-classes of elements of prime power order in the coset $x G$.

We will also use the following result of Steinberg [11].

Lemma 2.3. Let $x$ be an automorphism of the algebraic group $G$. Then $x$ normalizes some Borel subgroup of $G$.

We also need the following well-known easy result.

Lemma 2.4. Let $G$ be an algebraic group with $G^{0}=T$ a torus. Let $r$ be a positive integer. Then $G$ contains only finitely many conjugacy classes of elements of order $r$.

Proof. There is no harm in assuming that $G=\langle T, x\rangle$ with $x$ of order $r$. It suffices to show that the coset $x T$ contains only finitely many conjugacy classes of elements of order $r$. Let $V=[x, T]$. Then $V$ is a normal subtorus of $G$. If $V=T$, then all elements in $x T$ are conjugate, and so the result holds. If $V=1$, then $x$ commutes 
with $T$ and so $G=T \times\langle y\rangle$, where $y$ has order dividing $r$ and $G$ has only finitely many elements of order $r$.

If $V \neq 1$, then by induction, there are only finitely many conjugacy class of elements dividing $r$ in $G / V$, and so we may reduce to the case that $V=T$-a case already dealt with.

\section{The MAIN Results}

As in the previous section, we fix an algebraically closed field of characteristic $p$ and assume that $p>0$.

We first prove a result in the case of elements of order prime to $p$. In the first result, we do not need to assume that the group is reductive. The next two results (at least when the group is reductive) follow from the (equivalent) notions of strongly reductive (introduced by Richardson) and completely reducible subgroups (introduced by Serre). See [5] and [1].

Theorem 3.1. Let $S$ be an algebraic group of characteristic $p$ with $J=S^{0}$. Let $x \in S$ of order $r$ prime to $p$.

(1) $x$ normalizes a maximal torus $T$ of $S$.

(2) There are only finitely many conjugacy classes of elements of order $r$ in $S$.

Proof. By Lemma 2.3 any $x$ normalizes a Borel subgroup $B$ of $J$. So it suffices to prove the result for $S=\langle B, x\rangle$ (since all Borel subgroups are conjugate). Let $U$ be the unipotent radical of $B$.

We first prove (1). If $U$ is nonabelian, then we may pass to $S / Z(U)$, and by induction $x$ normalizes some conjugate of $T Z(U)$, and so a conjugate of $T$. If $U$ is abelian, then $V=[J, J]=[T, U]$ is normal in $S$. If $V=U$, then $T$ is the unique maximal torus of $T Z(U)$ and so $x$ normalizes it. If $V$ is proper, then by induction, we may assume that $x$ normalizes $T V$ and so a conjugate of $T$. This gives (1).

We now prove (2). Then every conjugacy class of elements of order $r$ has a representative in $N_{S}(T)$, and so it suffices to prove (2) when $T$ is normal in $S$, i.e. $J=T \times U$. By the previous result, we see that there are only finitely many conjugacy classes of order $r$ in $S / U$. On the other hand, since $r$ is prime to $p$, the coset $x U$ contains a unique conjugacy class of elements of order $r$.

In order to use induction, we need to know that centralizers of $p^{\prime}$-elements are reductive. This also follows from results in [1].

Lemma 3.2. Let $G$ be a reductive algebraic group and $x$ an automorphism of order $r$ prime to $p$. Then $C_{G}(x)$ is reductive.

Proof. Suppose not, and let $G$ be a counterexample of minimal dimension. There is no loss in assuming that $G$ is connected (since the centralizer of $x$ of the connected component has finite index in $\left.C_{G}(x)\right)$. So $G$ is a central product of a torus and a semisimple group. By minimality, we may assume that $G$ is either a torus or semisimple. If $G$ is a torus, so is the connected component of $C_{G}(x)$. So we may assume that $G$ is semisimple and that $x$ permutes the components of $G$ transitively. If there are $d$ components, then we see that $C_{G}(x) \cong C_{L}\left(x^{d}\right)$, where $L$ is a component. So we may assume that $d=1$. There is no harm in assuming that $x$ has prime order (for by induction on the order of $x$, the centralizer of $x^{e}$ is reductive, and we can consider $x$ acting on that). So either $x$ is inner and so $C_{G}(x)$ is the centralizer 
of a semisimple element and therefore reductive, or $x$ is a graph automorphism of order 2 or 3 . By inspection, $C_{G}(x)$ is reductive in these cases.

We next deal with unipotent elements and give an easy extension of the Spaltenstein result. Since we are in positive characteristic $p$, we say an element is unipotent if it is of order a power of $p$.

Theorem 3.3. Let $H$ be reductive. Then $H$ contains only finitely many conjugacy classes of unipotent elements.

Proof. We induct on $\operatorname{dim} H$. If $\operatorname{dim} H=0, H$ is finite, and the result is clear. Let $N$ be a minimal normal connected subgroup of $H$. So $N$ is reductive. The result is true for $H / N$ by induction. Thus, there are finitely many unipotent elements $u_{1}, \ldots, u_{t}$ such that $u N$ is conjugate to one of the $u_{i} N$ for any unipotent $u \in G$. So it suffices to prove the result for $H=\langle N, u\rangle$ with $u$ unipotent. Thus, we are reduced to the case that $N=H^{0}$. By the minimality of $N$, it follows that either $N$ is a central product of simple algebraic groups each isomorphic to $L$, a fixed simple algebraic group, or $N$ is a torus.

If $N$ is a torus, apply Theorem 3.1

If $N$ is semisimple, then passing to $H / Z(N)$ allows us to assume that $Z(N)=1$. Indeed, we may pass to $H / C_{H}(N)$, and so assume that $C_{H}(N)=1$ and that $u$ permutes transitively the $t$ components of $N$. So $t$ is a power of $p$. So $H$ is a finite index subgroup of $K:=\operatorname{Aut}(L) \prec \mathbb{Z} / t$. In this group, it is straightforward to compute that two elements $u, v$ inducing the same $t$-cycle on $N$ are conjugate if and only if $u^{t}$ and $v^{t}$ are conjugate. By Theorem 2.2. there are only finitely many conjugacy classes represented by elements $v^{t}$. So the result holds in $K$. Since $H$ has finite index in $K$, each conjugacy class of $K$ is a finite union of $H$-conjugates, whence the result holds.

We can combine the previous results to obtain:

Theorem 3.4. Let $H$ be reductive and $n$ a positive integer. Then $H$ contains only finitely many conjugacy classes of elements of order $n$.

Proof. We induct on $n$. If $n$ is a power of the characteristic, the previous result applies. So write $n=p^{a} n^{\prime}$, where $p$ does not divide $n^{\prime}$.

By the $p^{\prime}$-result, there are only finitely many conjugacy classes of elements of order $n^{\prime}$. If $y$ has order $n^{\prime}$, then its centralizer is reductive and so contains only finitely many conjugacy classes of unipotent elements, whence there are only finitely many conjugacy classes of elements of order $n$ with $p^{\prime}$-part $y$. This completes the proof.

We now can prove Theorem 1.1.

Theorem 3.5. Let $G$ be a reductive group. Let $C$ be a conjugacy class of $G$ and $n$ a positive integer. Then $D:=\left\{x \in G \mid x^{n} \in C\right\}$ is a finite union of conjugacy classes of $G$.

Proof. Fix $w \in C$. Let $N=G^{0}$ be the connected component of $G$. It suffices to show that the set of elements with $h^{n}=w$ intersects only finitely many conjugacy classes. If $w$ has finite order, the result follows from the previous result. If $w$ has infinite order, then $w^{d}$ is a nontrivial semisimple element of $N$ for some $d$. Then we may work in the reductive group $J:=C_{G}\left(w^{d}\right)$. If $\operatorname{dim} J<\operatorname{dim} G$, we are done 
by induction. If not, we see that $w$ has finite order modulo $Z(N)$, and since $w$ has infinite order, $Z(N)$ contains a nontrivial normal torus $T$. It now suffices to consider the case that $G=\langle T, h\rangle$ and as usual reduce to the abelian case, where the result is clear.

We now prove Theorem 1.2, Note that since we can always enlarge $G$, there would be no harm in assuming that $G$ is reductive or even simple in the next result.

Theorem 3.6. Let $G$ be an algebraic group and $H$ a reductive subgroup. If $x \in G$, then $x^{G} \cap H$ is a finite union of $H$-classes.

Proof. Choose an integer $m$ with the following properties (take $m$ to be a sufficiently large power of $p$ times the least common multiple of $\left[G: G^{0}\right]$ and $\left.\left[H: H^{0}\right]\right)$ :

(1) If $g \in G$, then $g^{m}$ is a semisimple element in the connected component of $G$; and

(2) if $y \in H$, then $y^{m}$ is a semisimple element in the connected component of $H$.

Set $y=x^{m}$. We first note that $y^{G} \cap H$ is a finite union of $H$-conjugacy classes. This follows since we may choose a maximal torus $S$ of $H$ contained in a maximal torus $T$ of $G$. Thus, it suffices to prove that $y^{G} \cap S$ is finite, and this follows since $y^{G} \cap T$ is an orbit under the normalizer of $T$, whence is finite.

So let $C_{i}, 1 \leq i \leq e$, be the $H$-classes contained in $y^{G} \cap H$. If $w \in x^{G} \cap H$, then $w^{m} \in y^{G} \cap H$, and so $w^{m} \in C_{i}$ for some $i$. As we have seen, the $m$ th roots of $C_{i}$ in $H$ are a finite union of $H$-classes, whence $x^{G} \cap H$ is a finite union of $H$-classes.

\section{REFERENCES}

[1] M. Bate, B. Martin and G. Röhrle, A geometric approach to complete reducibility, Invent. Math. 161 (2005), 177-218. MR2178661

[2] R. Lawther, Elements of specified order in simple algebraic groups, Trans. Amer. Math. Soc. 357 (2005), 221-245. MR2098093 (2005h:20104)

[3] G, Lusztig, On the finiteness of the number of unipotent classes. Invent. Math. 34 (1976), 201-213. MR0419635 (54:7653)

[4] G. Malle and K. Sorlin, Springer correspondence for disconnected groups, Math. Z. 246 (2004), 291-319. MR2031457 (2004k:20087)

[5] B. Martin, Reductive subgroups of reductive groups in nonzero characteristic, J. Alg. 262 (2003), 265-286. MR1971039 (2004g:20066)

[6] S. Murray, Conjugacy classes in maximal parabolic subgroups of general linear groups. J. Algebra 233 (2000), 135-155. MR1793594 (2002f:20069)

[7] R. W. Richardson, Conjugacy classes in Lie algebras and algebraic groups. Ann. of Math. 86 (1967), 1-15. MR0217079 (36:173)

[8] P. Slodowy, Two notes on a finiteness problem in the representation theory of finite groups. With an appendix by G.-Martin Cram. Austral. Math. Soc. Lect. Ser., 9, Algebraic groups and Lie groups, 331-348, Cambridge Univ. Press, Cambridge, 1997. MR1635690 (99e:20020)

[9] K. Sorlin, Springer correspondence in non connected reductive groups, J. Reine Angew. Math. 568 (2004), 197-234. MR2034927 (2005a:20066)

[10] N. Spaltenstein, Classes unipotentes et sous-groupes de Borel, Lecture Notes in Math. 946, Springer, Berlin, Heidelberg, New York, 1982. MR0672610 (84a:14024)

[11] R. Steinberg, Endomorphisms of linear algebraic groups, Memoirs of the Amer. Math. Soc. 80, Amer. Math. Soc., Providence, 1968. MR 0230728 (37:6288)

Department of Mathematics, University of Southern California, Los Angeles, CalIFORNIA 90089-2532

E-mail address: guralnic@usc.edu 